October 25, 2018 17:2 WSPC/Guidelines photo'070502

International Journal of Modern Physics B

(C) World Scientific Publishing Company

\title{
Phenomenological Modeling of Photoemission Spectra in Strongly Correlated Electron Systems
}

\author{
Krzysztof Byczuk, ${ }^{a}, b$ Ralf Bulla, ${ }^{a}$ Ralph Claessen,${ }^{c}$ and Dieter Vollhardt ${ }^{a}$ \\ (a) Theoretical Physics III, Center for Electronic Correlations and Magnetism, Institute of \\ Physics, University of Augsburg, D-86135 Augsburg, Germany \\ (b) Institute of Theoretical Physics, Warsaw University, ul. Hoża 69, 00-681 Warszawa, Poland \\ (c) Experimental Physics II, Institute of Physics, University of Augsburg, D-86135 Augsburg, \\ Germany \\ Received (received date) \\ Revised (revised date)
}

\begin{abstract}
A phenomenological approach is presented that allows one to model, and thereby interpret, photoemission spectra of strongly correlated electron systems. A simple analytical formula for the self-energy is proposed. This self-energy describes both coherent and incoherent parts of the spectrum (quasiparticle and Hubbard peaks, respectively). Free parameters in the expression are determined by fitting the density of states to experimental photoemission data. An explicit fitting is presented for the $\mathrm{La}_{1-x} \mathrm{Sr}_{x} \mathrm{TiO}_{3}$ system with $0.08 \leq x \leq 0.38$. In general, our phenomenological approach provides information on the effective mass, the Hubbard interaction, and the spectral weight distribution in different parts of the spectrum. Limitations of this approach are also discussed.
\end{abstract}

\section{Introduction}

Photoemission experiments provide important information about the electronic single-particle excitation spectrum of solids 1 For weakly correlated materials this is essentially given by the density of states (DOS) obtained by, e.g., density functional theory in combination with the local density approximation (LDA). 2 In many cases the agreement between LDA and experiment turns out to be very good. However, there is a class of materials where the discrepancy between the measured and cal-

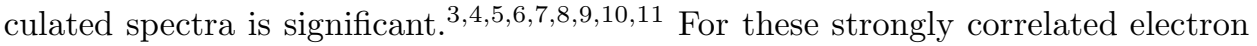
systems there is a clear demand for new theoretical and computational approaches. The recently developed LDA+DMFT method, a combination of the LDA and the dynamical mean field theory (DMFT), has proved to be very successful in this respect.12, 13, $14,15,16,17,18$ The LDA+DMFT method supplements the LDA by local correlations between $d$ - or $f$-electrons. 16 - 2 In the simplest case, namely, in the absence of long-range order and when the correlated bands at the Fermi level are sufficiently separated from other bands, 1618 the DOS of a correlated system is well 
represented by the integral (Hilbert transform)

$$
\rho_{\mathrm{LDA}+\operatorname{DMFT}}(\omega)=-\frac{1}{\pi} \operatorname{Im} \int d \omega^{\prime} \frac{\rho_{\mathrm{LDA}}\left(\omega^{\prime}\right)}{\omega-\omega^{\prime}-\Sigma_{\mathrm{DMFT}}(\omega)+i 0^{+}},
$$

where $\rho_{\mathrm{LDA}}(\omega)$ is the DOS from the LDA calculation, and $\Sigma_{\mathrm{DMFT}}(\omega)$ is the local self-energy calculated self-consistently within theDMFT scheme which includes correlation effects missing in the LDA approach 12 13, $44,15.16,47.28$ Non-local contributions to the self-energy cannot yet be implemented in this scheme. This will become possible in extensions of the DMFT, e.g., in the Dynamical Cluster Approximation (DCA) and related computational schemes. 19

The aim of this paper is to describe another approach, phenomenological in nature, to model photoemission spectra of strongly correlated electrons. The motivation is the following: the LDA+DMFT method is microscopic but requires an extensive numerical effort to calculate $\rho_{\mathrm{LDA}}(\omega)$ and $\Sigma_{\mathrm{DMFT}}(\omega)$. On the other hand, an analysis of various models of strongly correlated electrons within the DMFT has shown that certain features of the self-energy do not depend on the details of the model. Fermi liquid behaviour is seen in, e.g., Numerical Renormalization Group (NRG) calculations for the Hubbard model both at and away from half-filling (see Fio (2) below) and for the Periodic Anderson Model in the heavy-fermion regime 2121 In these systems, the imaginary part of the self-energy consists of the $\omega^{2}$-dependence for $\omega \rightarrow 0$ and $T \rightarrow 0$ and two pronounced peaks at finite $\omega$. On increasing the temperature, Fermi liquid behaviour can be rapidly destroyed, in particular close to the Mott transition, 22 or in general for systems with a very high effective mass. The imaginary part of the self-energy then goes over to a single and very broad peak centered approximately at the Fermi level (see, e.g., Fig. 5 in Ref.[22]). This model-independence of the self-energy suggests the use of a universal form for $\Sigma(\omega)$ which depends only on a small number of phenomenological parameters. This $\Sigma(\omega)$ replaces $\Sigma_{\mathrm{DMFT}}(\omega)$ in Eq. (11).

Although the proposed scheme is phenomenological (the parameters in the selfenergy being determined by fitting to the experimental data) we believe it to be useful for the qualitative interpretation and understanding of the experimental results. The phenomenological self-energy $\Sigma(\omega)$ obtained in this way can be used to deduce other quantities for the specific material, such as a linear specific heat coefficient and the dynamical conductivity (under the assumption that vertex corrections are negligible). This approach would then serve as a unifying phenomenological description of a variety of experimental results.

Conceptually, such an approach is not new. It was used previously to fit and interpret, for example, the integrated photoemission data for $\mathrm{Ca}_{1-x} \mathrm{Sr}_{x} \mathrm{VO}_{2}$, , and the angular resolved photoemission data in prototype Fermi liquid metals 2324 and hightemperature superconductors. 25 However, only the quasiparticle peak was fitted in these approaches. Here we provide an analytical expression for the self-energy which is appropriate for fitting the whole spectrum of correlated $d$ - or $f$-electrons, where the Hubbard subbands and the quasiparticle resonance are present simultaneously. 
Our approach is based on a sum of Lorentz functions. Matho has proposed another route based on the multi-pole expansion of the phenomenological self-energy.26 It turns out that these two approaches are mathematically equivalent. In our approach it is possible to present a simple physical motivation for the form of the self-energy.

Our phenomenological approach encounters certain difficulties which should be mentioned here. The most serious problem is connected with the description of multi-band systems. The strong electronic correlations originate from the localized nature of $f$ - or $d$-orbitals. Hence, several bands might cross the Fermi level or be very close to it even when they are split by, e.g., a crystal field. In $\mathrm{V}_{2} \mathrm{O}_{3}$, for instance, the splitting between the $t_{2 g}$ and $e_{g}$ bands is rather small. In such a case, each band which lies in the vicinity of the Fermi level would require a separate self-energy, which makes the number of fitting parameters twice or three times larger. Since the photoemission results are not orbitally resolved, an unambiguous fitting cannot be guaranteed in these cases. Without additional experimental input, a phenomenological approach for these cases is not adequate.

In our paper we therefore concentrate on the experimental data for $\mathrm{La}_{1-x} \mathrm{Sr}_{x} \mathrm{TiO}_{3}$, a system with degenerate $t_{2 g}$ bands (see Sec. 4). Before that — in Secs. 2 and $3-$ the phenomenological expression for $\Sigma(\omega)$ is introduced. The results of our paper are summarized in Sec. 5 .

\section{Self-Energy}

We start with a heuristic derivation of the retarded self-energy for strongly correlated electrons (e.g., $d$-electrons) in the metallic phase which form a Fermi liquid state at low energies and temperatures. The DOS $\rho(\omega)$, calculated with this selfenergy should consist of three parts: two wide incoherent parts (upper and lower Hubbard bands) and a coherent peak at or close to the Fermi level $\mu$.

The construction of a suitable self-energy expression is guided by the following idea. Let us start with a model for the spectral function $A_{\bmod }(\omega)$ which is a sum of three Lorentz curves

$$
\begin{gathered}
A_{\bmod }(\omega)=\frac{Q}{\pi} \frac{\gamma}{\left(\omega-\omega_{0}\right)^{2}+\gamma^{2}}+ \\
\frac{(1-Q)}{\pi}\left[\frac{q}{2} \frac{\Gamma}{\left(\omega-\frac{I}{2}\right)^{2}+\Gamma^{2}}+\left(1-\frac{q}{2}\right) \frac{\Gamma}{\left(\omega+\frac{I}{2}\right)^{2}+\Gamma^{2}}\right] .
\end{gathered}
$$

One peak (the quasiparticle peak) is centered at $\omega=\omega_{0}$ with spectral weight $Q \geq 0$ and width $\gamma \geq 0$. The other two peaks (upper and lower Hubbard peaks) are centered at $\omega= \pm I / 2$ and their widths are assumed to be both equal to $\Gamma \geq 0$ (see Fig. (11)). The total weight of these two peaks is $1-Q$ with the relative weights $q / 2 \geq 0$ and $1-q / 2 \geq 0$ respectively. $A_{\bmod }(\omega)$ is normalized to unity. Apart from assuming the same width for both upper and lower Hubbard peaks, Eq. (2) is the most general sum of three Lorentz peaks. Since we have not yet specified 
4 K. Byczuk, R. Bulla, R. Claessen, D. Vollhardt

the position of the chemical potential $\mu$, Eq. (2) describes both symmetric and asymmetric cases.

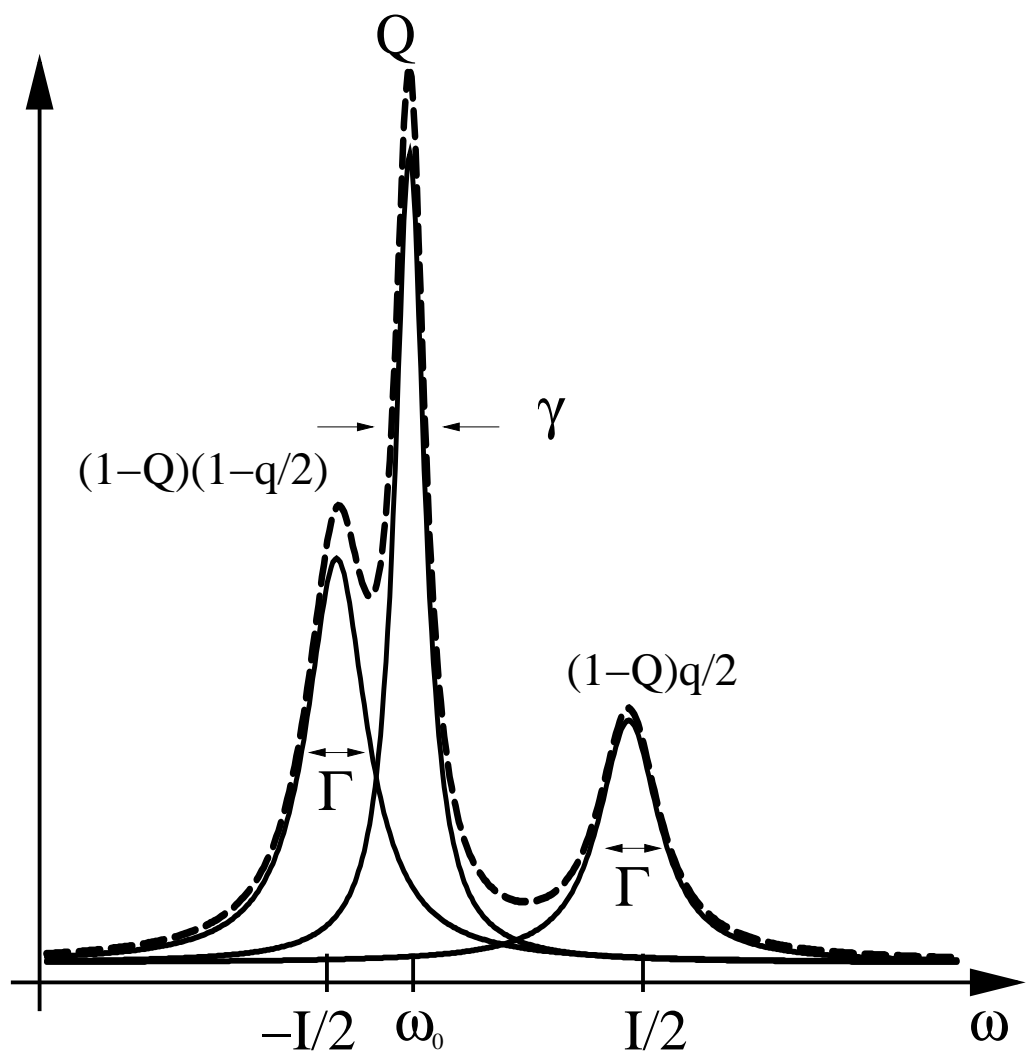

Fig. 1. Schematic plot of the three peaks of the model spectral function $A_{\bmod }(\omega)$.

There is, of course, some arbitrariness in the choice of Eq. (2) for $A_{\bmod }(\omega)$. Lorentzians are used here to obtain simple analytical expressions. One can try to use, for example, Gauss or semi-elliptic forms of the DOS as well. However, in these cases $\Sigma(\omega)$ either cannot be expressed analytically or is not a smooth function of $\omega$.

The retarded local Green function $g_{\text {mod }}(\omega)$ corresponding to the spectral function (2) is

$$
g_{\bmod }(\omega)=\frac{Q}{\omega-\omega_{0}+i \gamma}+(1-Q)\left[\frac{q}{2} \frac{1}{\omega-\frac{I}{2}+i \Gamma}+\left(1-\frac{q}{2}\right) \frac{1}{\omega+\frac{I}{2}+i \Gamma}\right] .
$$


We also introduce a Green function

$$
g_{\text {mod }}^{0}(\omega)=\frac{1}{\omega-\omega_{1}+i \Delta},
$$

which corresponds to a spectral function $A_{\text {mod }}^{0}(\omega)$ with a single peak centered at $\omega=\omega_{1}$ and with the width $\Delta \geq 0$. Note that all the above quantities (i.e. those with an index 'mod') do not correspond to any physical quantity but are introduced for the construction of a suitable self-energy only.

Using the Green functions (3) and (4) together with the Dyson equation for the self-energy $\Sigma_{\text {mod }}(\omega)=g_{\text {mod }}^{0}(\omega)^{-1}-g_{\text {mod }}(\omega)^{-1}$ we find

$$
\Sigma_{\text {mod }}(\omega)=\omega-\omega_{1}+i \Delta-\left[\frac{Q}{\omega-\omega_{0}+i \gamma}+(1-Q) \frac{\omega+i \Gamma-(1-q) \frac{I}{2}}{(\omega+i \Gamma)^{2}-\left(\frac{I}{2}\right)^{2}}\right]^{-1} .
$$

This self-energy contains 8 parameters. The number of parameters may be reduced by imposing additional conditions which are discussed below.

In order to preserve the Fermi liquid properties at low energy we have to supplement the self-energy (5) by the condition $\operatorname{Im} \Sigma(\omega=\mu)=0.27$ Then, as $\omega \rightarrow 0, \operatorname{Re} \Sigma(\omega) \sim-\omega$, and $\operatorname{Im} \Sigma(\omega) \sim-\omega^{2}$. In the high energy limit $\omega \rightarrow \infty$, $\operatorname{Re} \Sigma(\omega) \sim 1 / \omega$, but $\operatorname{Im} \Sigma(\omega) \sim a-1 / \omega^{2}$ with a constant $a \geq 0$, which means that the imaginary part of the self-energy $\Sigma(\omega)$ changes sign. We have checked that this artefact becomes important only if $I$ is much smaller than $\gamma$. However, in this weakly correlated limit $\rho_{\mathrm{LDA}}$ usually reproduces the experimental data reliably and the corrections due to $\Sigma(\omega)$ are not necessary. In the strongly correlated limit the change of the sign in $\operatorname{Im} \Sigma(\omega)$ appears at high energies. We have therefore introduce a cut-off setting $\operatorname{Im} \Sigma(\omega)=0$ whenever the self-energy becomes positive.

The self-energy (5) is temperature independent. As noted in the introduction, the self-energy develops a peak at the chemical potential $\mu$ for finite temperature, in particular close to the Mott transition. This effect is described phenomenologically by introducing a scattering part

$$
\Sigma_{\text {scatt }}(\omega)=\frac{s}{\omega-\mu+i \gamma_{s}},
$$

with two fitting parameters $s$ and $\gamma_{s}$. The scattering part is not used in this paper but it might be important in systems far away from the Fermi liquid regime.

Hence, the phenomenological self-energy takes the form

$$
\Sigma_{\mathrm{fit}}(\omega)=\Sigma_{\bmod }(\omega)+\Sigma_{\text {scatt }}(\omega) .
$$

Before applying Eq. (7) to model experimental data, we check whether this form of the self-energy can reproduce the self-energies obtained numerically from the DMFT equations of the Hubbard model

$$
H=-t \sum_{<i j>\sigma}\left(c_{i \sigma}^{\dagger} c_{j \sigma}+c_{j \sigma}^{\dagger} c_{i \sigma}\right)+U \sum_{i} c_{i \uparrow}^{\dagger} c_{i \uparrow} c_{i \downarrow}^{\dagger} c_{i \downarrow},
$$

where $t$ is the hopping matrix element between nearest neighbour sites and $U$ is the local interaction energy for the electrons with antiparallel spins $\sigma$. Figure 2 
shows the result of the fit to the spectral function, calculated by NRG with the microscopic parameters $U=4, \mu=-1.4$ and $T=0$. We imposed the Fermi liquid condition $\Sigma_{\text {fit }}(\omega=\mu)=0$. The scattering part $\Sigma_{\text {scatt }}(\omega)$ was set to zero. The parameters determined from the fit-procedure are $\omega_{0}=0.02, \delta=0.005, \gamma=0.005$, $\Gamma=0.51, Q=0.3, q=0.72$, and $I=4.5$. We used the same bare DOS as in the NRG calculation, i.e. a semielliptic DOS with the width $W=2$. This width sets the energy units in this fitting. The comparison shows both the possibilities and limitations of our phenomenological approach. Although the three-peak structure and the overall distribution of the spectral weight are described correctly, there are significant deviations regarding the form of the peaks. The main reason for this is that the peaks in the numerical data are not Lorentzian (see, e.g., the discussion of the form of the Kondo resonance in the single impurity Anderson model in [28]). The fit-procedure therefore cannot recover the dip at $\omega \approx 1.5$, and compensates this by underestimating the width of the upper Hubbard peak. Also, the band filling determined from the fitted spectral function is larger by about $7 \%$ as compared to the NRG result.

The same holds for the structures obtained for the self-energy as is seen in the lower panel of Fig. (2). These are not, according to the NRG result, given by Lorentz peaks. Nevertheless, the general structure, i.e., the Fermi-liquid behavior for small frequencies and the two peaks at higher frequencies $(\omega-\mu \approx-0.5$ and 2.8) is reproduced correctly. The relative difference in the weight of these latter peaks is due to the particle-hole asymmetry in the parameters used for this particular calculation.

\section{Modeling Photoemission Spectra}

The phenomenological form for the self-energy Eq.(17) discussed in the previous section can now be inserted in the Hilbert transformation for the DOS

$$
\rho_{\text {fit }}(\omega)=-\frac{1}{\pi} \operatorname{Im} \int d \omega^{\prime} \frac{\rho_{\mathrm{LDA}}\left(\omega^{\prime}\right)}{\omega-\omega^{\prime}-\Sigma_{\mathrm{fit}}(\omega)+i 0^{+}} .
$$

The direct $\left(S_{\text {direct }}\right)$ and inverse $\left(S_{\text {inverse }}\right)$ photoemission intensities, within a constant transfer matrix approximation, are given by

$$
\begin{gathered}
S_{\text {direct }}(\omega)=S_{0} \int_{-\infty}^{\infty} d \omega^{\prime} R_{\sigma}\left(\omega-\omega^{\prime}\right) f\left(\frac{\omega^{\prime}-\mu}{k T}\right) \rho_{\text {fit }}\left(\omega^{\prime}\right), \\
S_{\text {inverse }}(\omega)=S_{0}^{\prime} \int_{-\infty}^{\infty} d \omega^{\prime} R_{\sigma^{\prime}}\left(\omega-\omega^{\prime}\right)\left[1-f\left(\frac{\omega^{\prime}-\mu}{k T}\right)\right] \rho_{\text {fit }}\left(\omega^{\prime}\right),
\end{gathered}
$$

where $f\left[(\omega-\mu) / k_{\mathrm{B}} T\right]$ is the Fermi-Dirac function with the chemical potential $\mu$ at the temperature $k_{\mathrm{B}} T$ (in energy units) and $R_{\sigma}(\omega)=\exp \left(-\omega^{2} / 2 \sigma^{2}\right) / \sqrt{2 \pi} \sigma$ is the apparatus function with the resolution $\sigma$. Note that the resolution in direct photoemission experiments is typically about one or two orders of magnitude better than in inverse photoemission experiments. $S_{0}$ and $S_{0}^{\prime}$ are constant prefactors. 


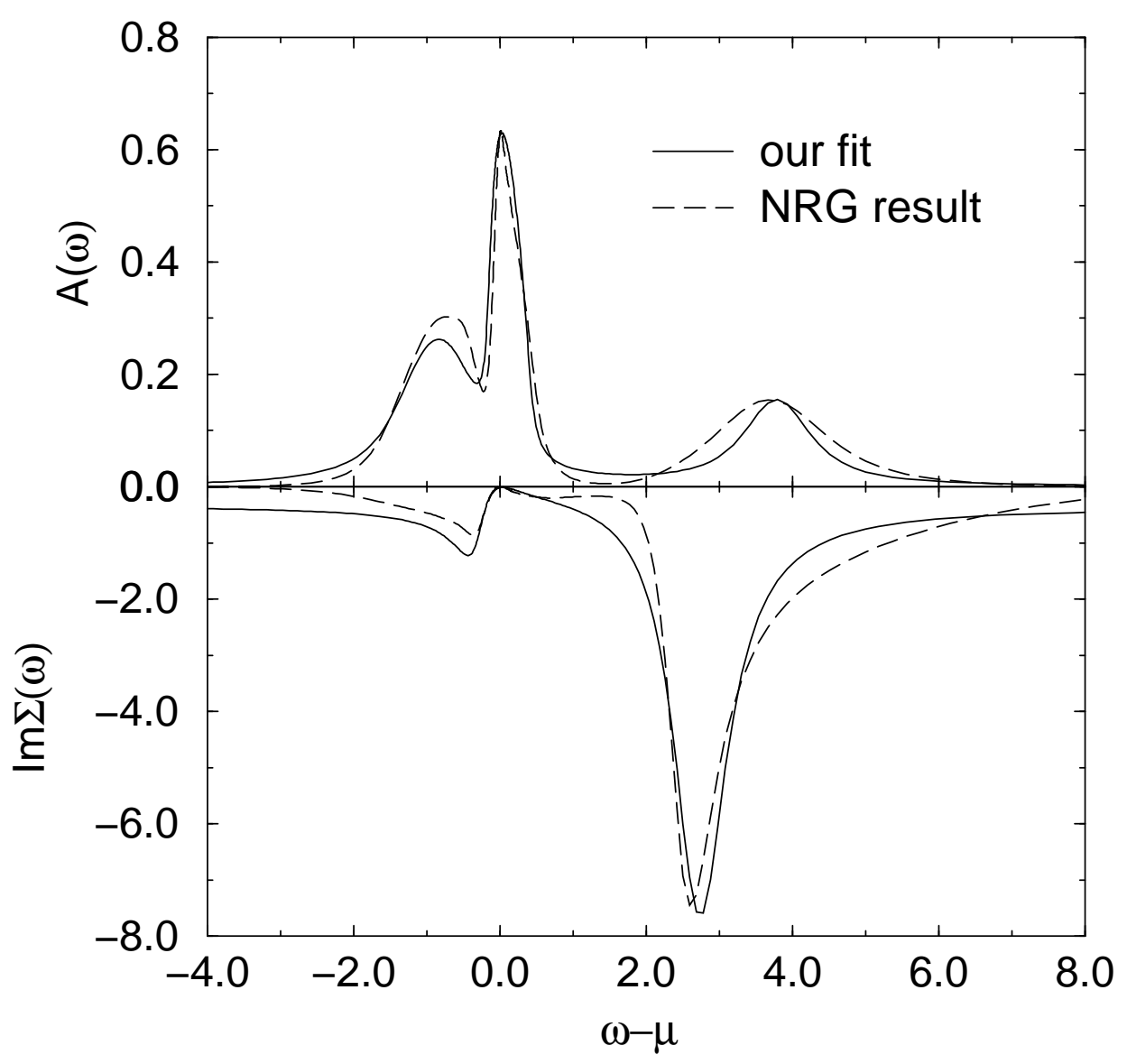

Fig. 2. Upper panel: The fitted spectral function using the self-energy (5) (solid line) and the numerical spectra from NRG (dashed line). Lower panel: The phenomenological self-energy corresponding to the fitting in the upper panel (solid line) and the self-energy from NRG (dashed line). Scales on the horizontal axis are the same.

With good quality data for the direct and the inverse photoemission spectra on the same sample under the same conditions one can now determine the phenomenological parameters in the self-energy (7) and determine, for example, the effective mass, the magnitude of the Hubbard interaction and the full frequency dependence of the self-energy.

\section{Example of Fitting}

We now turn to the phenomenological modeling of experimental photoemission data, as exemplified in the case of $\mathrm{La}_{1-x} \mathrm{Sr}_{x} \mathrm{TiO}_{3}$. In this compound, the $3 d^{1}$ electrons occupy degenerate $t_{2 g}$ orbitals for which the crystal and Jahn-Teller splittings are very small. Moreover, the $t_{2 g}$ band is well separated from the $e_{g}$ and $p$-bands. 
Our phenomenological modeling can therefore be restricted to a single degenerate band.

The available direct photoemission spectral show the typical features of a strongly correlated metal, i.e., a quasiparticle peak and a lower Hubbard band. At low temperature $\mathrm{La}_{1-x} \mathrm{Sr}_{x} \mathrm{TiO}_{3}$ is an antiferromagnetic insulator for $x=0$ and a band insulator with an empty $d$-band for $x=1$. Consequently, the filling of the threefold degenerate $d$-band decreases from $n=1$ to $n=0$ in going from $x=0$ to $x=1$. The antiferromagnetic insulator is stable up to $x \approx 0.05$ for $T<T_{N}\left(T_{N}=112 \mathrm{~K}\right.$ for $x=0$ ), and for $0.05<x<0.08$ an antiferromagnetic metallic phase appears with decreasing $T_{N}$ with increasing $x$.

In the photoemission measurement, the quasiparticle peak is clearly visible in Fig. (3) for all paramagnetic samples with $x=0.08,0.18,0.28,0.35$. herent peak is suppressed in the antiferromagnetic metallic phase $(x=0.06)$, and vanishes in the insulating regime $(x=0.04)$. The wide incoherent peak - the lower Hubbard band - is present for all $x$-values. These features, in particular the lower Hubbard band, cannot be explained within the LDA approach.29

Here we have used the experimental data to determine the phenomenological parameters in the self-energy (7). However, since we only know the occupied part of the spectrum it is impossible to determine unambiguously the absolute value of $I$, corresponding to the distance between the lower (occupied) and the upper (unoccupied) Hubbard bands. Only a relative value $I / 2+\mu$ can be determined. In order to make the problem tractable we have to reduce the number of parameters.

To this end we set $I=5 \mathrm{eV}$ since this is the value found in other theoretical studies. 0 Also we assume that the $t_{2 g}$ band is three-fold degenerate and that $x=1$ corresponds to $1 / 3$ filling. With decreasing $x$ the filling of this band is lowered and is found to be $n=(1-x) / 3$, after normalizing the DOS to unity. This filling for a given $x$ is used as another constraint on the parameters in our self-energy (7). In other words, the parameters are adjusted such as to obtain the correct filling $n=(1-x) / 3$ of the $d$-band. We do not use the Fermi liquid constraint because the experiment was performed at finite temperature and close to the Mott transition, so that deviations from $\operatorname{Im} \Sigma(\omega=0)=0$ are expected to be significant.

Within these assumptions the best fit is obtained by minimizing the mean square deviation between the theoretical and experimental data. The apparatus resolution function is taken to be a Gauss function with the variance $\sigma=0.035 \mathrm{eV}$ and the temperature is $T=20 \mathrm{~K}$. The bare DOS is used as for $\mathrm{LaTiO}_{3}$ from Ref.[29]. One example is shown in the lower panel of Fig. (3).

For comparison, we have also fitted the coherent part of the spectrum using the phenomenological self-energy

$$
\Sigma_{\mathrm{QP}}(\omega)=g \frac{a \omega}{\omega+i a} \cdot \frac{b}{\omega+i b} .
$$

This form of $\Sigma(\omega)$ is useful to fit the quasiparticle peak but does not describe the Hubbard bands. In situations with no clear separation of these two structures, a 


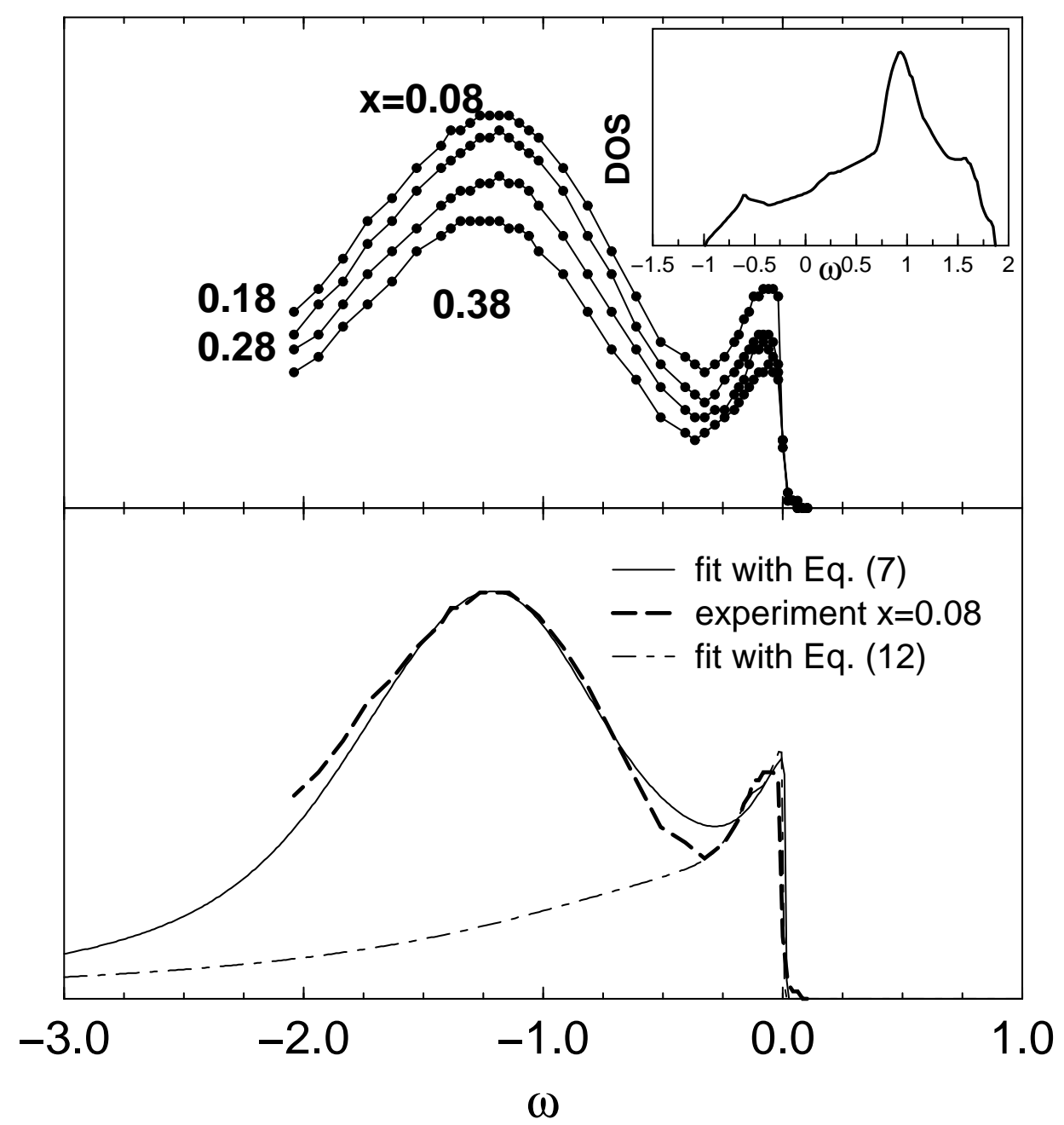

Fig. 3. Upper panel: Four curves from the photoemission experiments 9 for $\mathrm{La}_{1-x} \mathrm{Sr}_{x} \mathrm{TiO}_{3}$. Black dots show digitized points and small wiggles are due to digitization errors. The inset shows the DOS from LDA calculations for this system 29 Lower panel: Example of fitting the self-energy (17) (solid line) and the self-energy (12) (dot-dashed line) to the experimental result (dashed thick line) with $x=0.08$.

fit using Eq.(12) obviously involves some arbitrariness. This is in contrast to our fit formula for the self-energy which does not require the two structures to be well separated.

As we can see in Fig. (3) both self-energies (7) and (12) provide a similar description of the quasiparticle peak. However, the self-energy (7) also fits the high-energy feature of the spectrum. In Fig. (画) we show the total spectral functions (upper 


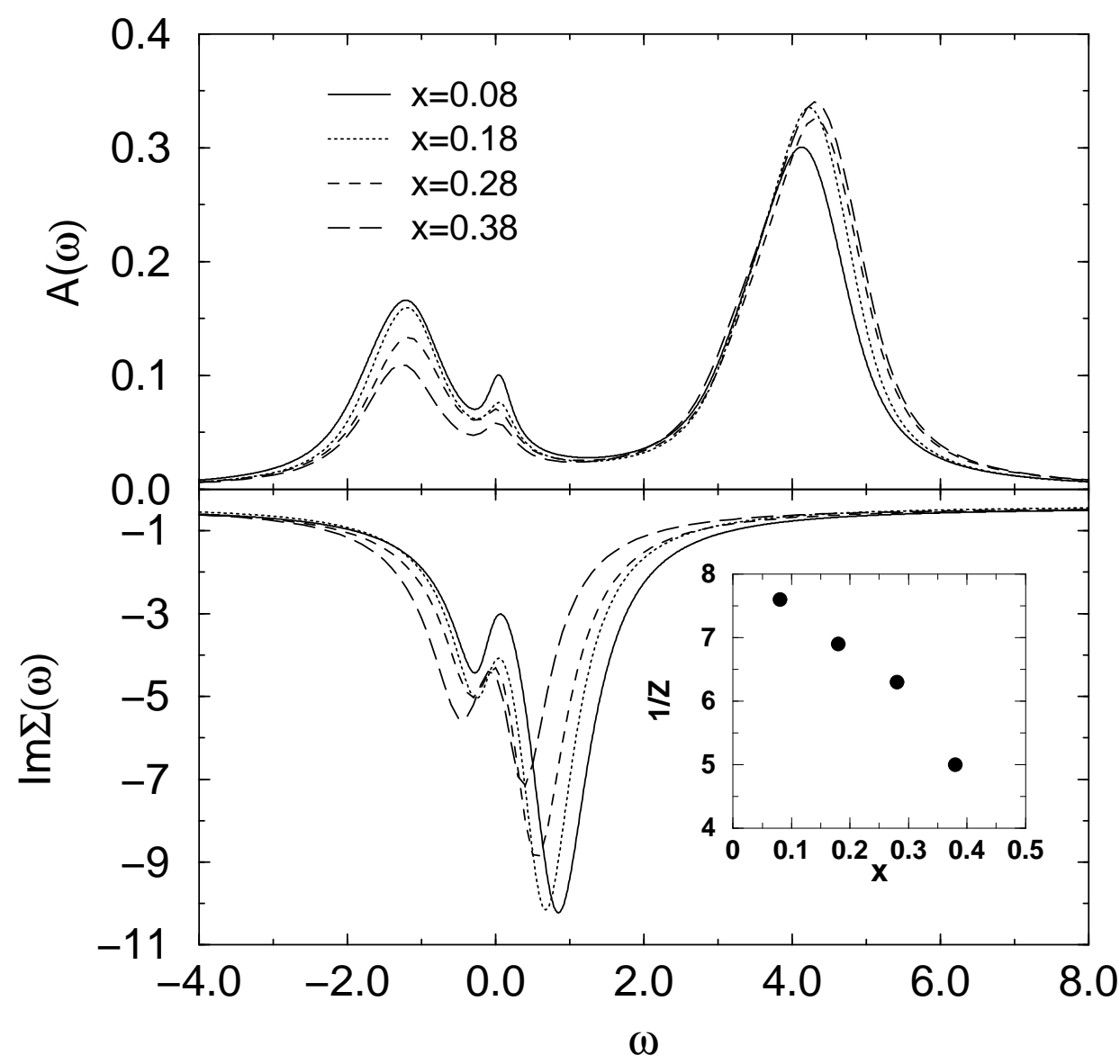

Fig. 4. Upper panel: The spectral functions for different $x$ obtained from fitting to experimental data, as described in the text. Lower panel: Imaginary part of the self-energy for different $x$. The inset shows the behavior of $1 / Z$, extracted from the real part of the self-energy, as a function of $x$.

panel) for four different values of $x$ obtained with this procedure. The lower panel presents the imaginary parts of the self-energy (5). As we can see, with decreasing $x$ the number of states below the Fermi level is reduced and more spectral weight is pushed into the upper Hubbard peak. Furthermore, the quasiparticle peak is suppressed for higher $x$. These trends are also mirrored in the behavior of the selfenergy for different $x$. The absolute value of $\operatorname{Im} \Sigma$ at the Fermi level increases with $x$. This increase might be attributed to the enhanced scattering due to the randomness introduced by the $\mathrm{Sr}$ atoms in the system. We also calculated the $Z$-factor $(1 / Z=1-\partial \operatorname{Re} \Sigma(\omega=\mu) / \partial \omega)$ which in Fermi liquid theory is related to the effective mass of the quasiparticles $\left(1 / Z \sim m^{\star} / m\right)$. In the inset to Fig. (四) we see a reduction of $1 / Z$ with increasing $x$. 
From these results, obtained phenomenologically, we are able to conclude that $\mathrm{La}_{1-x} \mathrm{Sr}_{x} \mathrm{TiO}_{3}$ for $x>0.06$ is a correlated metal which can be modeled with a local self-energy. The origin of the narrow quasiparticle peak and the lower Hubbard band can only be explained within a microscopic approach, e.g., DMFT. This is indeed possible, as has been shown recently using the LDA+DMFT approach.15

The $Z$-factor determined above is of the same order for both forms of the phenomenological self-energy [(7) and (12)]. Nevertheless, we find that the particular value of $Z$ depends on the precise form of $\Sigma(\omega)$. The dependence of $Z$ on $x$ also turns out to be different for the two self-energies. Using Eq. (5) its behavior is shown in the inset to Fig. (何. From Eq. (12) we obtained values between 3.5 to 4 for the corresponding $x$. This is because the phenomenological self-energy is used to fit the spectrum in a finite frequency window around $\omega=\mu$, which might be much larger then the actual frequency window for which Fermi liquid theory is valid. One should therefore be cautious in the interpretation of the actual values obtained for the effective mass $m^{\star}$.

We also note an intriguing feature of the experimental data for $x>0.06 .9$ When the curves are normalized to unity and plotted in a single figure, they are almost identical. In particular, the distance between the coherent peak and the top of the Hubbard band does not depend on $x$. This is in striking contrast to numerical calculations for a doped Hubbard model where doping inevitably leads to a shift of the chemical potential towards the lower Hubbard band (at least under the assumption of a homogeneous phase). Such a discrepancy has recently also been observed for the related compound $\mathrm{Gd}_{1-x} \mathrm{Sr}_{x} \mathrm{TiO}_{3}$ where it was attributed to an inhomogeneous sample composition due a chemical phase separation into strongly and poorly doped domains. 30 Such a phase separation may be restricted to the surface, as bulk-sensitive experiments did not observe it. A possible difference between surface and bulk electronic structure in oxidic perovskites has indeed been reported for doped vanadates 31.62

\section{Conclusions and Final Remarks}

In this paper we presented a simple analytical expression (5) for the self-energy which can be used to fit experimental data for strongly correlated electron systems. As an application we presented fits to the photoemission data for $\mathrm{La}_{1-x} \mathrm{Sr}_{x} \mathrm{TiO}_{3}$, for which the phenomenological fit parameters all take reasonable values.

There are certain difficulties in carrying out such a program, mainly due to the limited spectroscopic information which is currently available for correlated electron systems. Direct and inverse photoemission data would help to enhance the quality of the fits, in particular, if they allow one to fit different bands. Angular resolved photoemission experiments would give further information, and could provide insights into the validity of assuming a purely local self-energy.

At the moment, our approach is particularly useful to fit data for systems with partially filled, degenerate bands which are well separated from other completely 
filled or empty bands as, e.g., in $\mathrm{La}_{1-x} \mathrm{Sr}_{x} \mathrm{TiO}_{3}$.

\section{Acknowledgements}

It is a pleasure to acknowledge M. Potthoff for discussions. This research was support in part by the Deutsche Forschungsgemeinschaft through the Sonderforschungsbereich 484. The work of K.B. was sponsored by the Alexander von Humboldt foundation through their scholarship program.

\section{References}

1. S. Hüfner, Photoemission Spectroscopy (Springer, Berlin, 1995).

2. R.O. Jones, O. Gunnarsson, Rev. Mod. Phys. 61, 689 (1989).

3. A. Fujimori, I. Hase, H. Namatame, Y. Fujishima, Y. Tokura, H. Eisaki, S. Uchida, K. Takegahara and F.M.F. de Grot, Phys. Rev. Lett. 69, 1769 (1992); Phys. Rev. B 46, 9841 (1992).

4. S.W. Robery, L.T. Hudson, C. Eylem and B. Eichorn, Phys. Rev. B 48, 562 (1993).

5. I.H. Inoue, I. Hase, Y. Aiura, A. Fujimori, Y. Haruyama, T. Maruyama and Y. Nishihara, Phys. Rev. Lett. 74, 2539 (1995).

6. K. Morikawa, T. Mizokawa, K. Kobayashi, A. Fujimori, H. Eisaki, S. Uchida, F. Iga and Y. Nishihara, Phys. Rev. B 52, 13711 (1995).

7. K. Morikawa, T. Nizokawa, A. Fujimori, Y. Taguchi and Y. Tokura, Phys. Rev. B 54, 8446 (1996).

8. H. Kim, H. Kumigashira, A. Ashihara and T. Takahashi, Phys. Rev. B 57, 1316 (1998).

9. T. Yoshida, A. Ino, T. Mizokawa, A. Fujimori, Y. Taguchi, T. Katsufuji and Y. Tokura, cond-mat/9911446 (unpublished); A. Fujimori, T. Yoshida, K. Okazaki, T. Tsujioka, K. Kobayashi, T. Mizokawa, M. Onoda, T. Katsufuji, Y. Taguchi, Y. Tokura, J. of Electron Spectroscopy and Related Phenomena 117-118, 277 (2001).

10. M. Schramme, Ph. D. Thesis - Augsburg University (2000).

11. Hyeong-Do Kim, J.-H. Park, J. W. Allen, A. Sekiyama, A. Yamasaki, K. Kadono, S. Suga, Y. Saitoh, T. Muro, P. Metcalf, cond-mat/0108044 (unpublished).

12. V.I. Anisimov, A.I. Poteryaev, M.A. Korotin, A.O. Anokhin, G. Kotliar, J. Phys.: Condens. Matt. 9, 7359 (1997).

13. A.I. Lichtenstein, M.I. Katsnelson, Phys. Rev. B 57, 6884 (1998).

14. M.B. Zölfl, Th. Pruschke, J. Keller, A.I. Poteryaev, I.A. Nekrasov, V.I. Anisimov, Phys. Rev. B61, 6884 (2000).

15. I.A. Nekrasov, K. Held, N. Blümer, A.I. Poteryaev, V.I. Anisimov, and D. Vollhardt, Euro. Phys. J. B 18, 55 (2000).

16. K. Held, I.A. Nekrasov, N. Blümer, V.I. Anisimov, and D. Vollhardt, Int. J. Mod. Phys. B 15, 2611 (2001).

17. K. Held, G. Keller, V. Eyert, D. Vollhardt, and V.I. Anisimov, Phys. Rev. Lett. 86, 5345 (2001).

18. K. Held, I.A. Nekrasov, G. Keller, V. Eyert, N. Blümer, A.K. McMahan, R.T. Scalettar, T.Pruschke, V.I. Anisimov, and D. Vollhardt, in Quantum Simulations of Complex Many-Body Systems: From Theory to Algorithms, eds. J. Grotendorst, D. Marx and A. Muramatsu, NIC Series Vol. 10 (NIC Directors, Forschungszentrum Jülich, 2002), p. 175 .

19. M. Jarrell and H.R. Krishnamurthy, Phys. Rev. B 63, 125102 (2001); M. Jarrell, Th. Maier, M. H. Hettler, A.N. Tahvildarzadeh, Euro. Phy. Letters 56, 563 (2001); M. Jarrell, Th. Maier, C. Huscroft, and S. Moukouri, Phys. Rev. B 64, 195130 (2001); G. 
October 25, 2018 17:2 WSPC/Guidelines photo*070502

Phenomenological Modeling of Photoemission Spectra in Strongly Correlated Electron Systems

Biroli and G. Kotliar, Phys. Rev. B 65, 155112 (2002); G. Kotliar, S. Y. Savrasov, G. Pálsson, and G. Biroli, Phys. Rev. Lett. 87, 186401 (2001).

20. R. Bulla, Phys. Rev. Lett. 84, 136 (1999).

21. Th. Pruschke, R. Bulla, and M. Jarrell, Phys. Rev. B 61, 12799 (2000).

22. R. Bulla, T.A. Costi, and D. Vollhardt, Phys. Rev. B 64, 045103 (2001).

23. R. Claessen, R.O. Anderson, J.W. Allen, C.G. Olson, C. Janowitz, W.P. Ellis, S. Harm, M. Kalning, R. Manzke, and M. Skibowski, Phys. Rev. Lett. 69, 808 (1992).

24. J.W. Allen, G.-H. Gweon, R. Claessen, and K. Matho, J. Phys. Chem. Solids 56, 1849 (1995).

25. See e.g. M.R. Norman, M. Randeria, H. Ding, J. C. Campuzano, Phys. Rev. B 57, 11093 (1998).

26. K. Matho, Molecular Phys. Reports 17, 141 (1997); J. Electron Spectroscopy and Related Phenomena 117-118, 13 (2001).

27. The equation expressing the Fermi liquid constraint is quite lengthly and not printed here.

28. R. Bulla, M.T. Glossop, D.E. Logan, and Th. Pruschke, J. Phys.: Condens. Matter 12, 4899 (2000).

29. K. Takegahara, J. of Electron Spectroscopy and Related Phenomena 66, 303 (1994).

30. M. Sing, M. Karlsson, D. Schrupp, R. Claessen, M. Heinrich, V. Fritsch, H.-A. Krug von Nidda, A. Loidl, R. Bulla, cond-mat/0205067 (unpublished).

31. K. Maiti, D.D. Sarma, M.J. Rosenberg, I.H. Inoue, H. Makino, O. Goto, M. Pedio, and R. Cimino, Euro. Phys. Lett. 55, 246(2001).

32. S. Suga and A. Sekiyama, private communication. 